\title{
An update on the prevention of influenza in children and adolescents
}

Received: 10 July 2003 / Revised: 11 August 2003 / Accepted: 21 August 2003/Published online: 21 October 2003

(C) Springer-Verlag 2003

\begin{abstract}
Influenza virus types A and B cause yearly outbreaks of respiratory tract infections in all age groups including children and adolescents. Complications, such as high fever, febrile convulsions, secondary bacterial infections and myositis frequently lead to hospitalisation. Safe and effective split, subunit and virosome vaccines are available from 6 months of age onwards. Most European countries do have guidelines for the use of influenza vaccines and current strategies primarily aim at decreasing the burden of influenza disease in certain, heterogeneously defined high risk groups. Conclusion: Unfortunately, compliance of many physicians and patients with immunisation recommendations is rather poor and several barriers to immunisation have been identified. These deserve our specific attention in the future. Recently, neuraminidase inhibitors with curative and preventive efficacy against influenza virus types A and B have become available. They serve as second line weapons for influenza prophylaxis under specific circumstances.
\end{abstract}

Keywords Immunization Influenza - Neuraminidase inhibitors $\cdot$ Prevention $\cdot$ Review

Abbreviations $C A I V$ - $T$ cold-adapted trivalent influenza vaccine $\cdot H A$ haemagglutinin $\cdot N A$ neuraminidase $\cdot$ $N I$ neuraminidase inhibitor $\cdot R S V$ respiratory syncytial virus

The author has temporarily served on the "Data Monitoring Safety Board" for an intranasal influenza vaccine (Nasalflu) of Berna Biotech Ltd, Bern, Switzerland and has actively participated as an investigator in pre-licensure studies of influenza vaccines produced by Berna Biotech and Wyeth.

U. Heininger

University Children's Hospital (UKBB), PO Box, 4005 Basel,

Switzerland

E-mail: Ulrich.Heininger@unibas.ch

Tel.: + 41-61-6856565

Fax: + 41-61-6856012

\section{Introduction}

Although immunisation against influenza has been recommended for patients with underlying chronic diseases for several decades in Europe and other parts of the world, vaccine up-take in risk groups has remained low $[9,25,39]$. This is probably due to several immunisation barriers which have been identified among physicians and patients $[5,6,23,58,59]$. Most importantly, misconceptions on the true burden of disease and its complications and lack of awareness of the benefits of influenza prevention can be blamed for this unfortunate state of affairs [23, 40, 55,62].

Traditionally, influenza has been considered a serious medical problem in elderly people whereas its significant impact on young infants, children and adolescents has, until recently, been widely neglected [14]. Here, I will briefly review the current knowledge on epidemiology and clinical characteristics of influenza in the paediatric age group. Efficient ways of prophylaxis, i.e. by immunisation and use of neuraminidase inhibitors (NIs) will be discussed with a specific focus on the situation in Europe. Moreover, potential interventions to improve vaccine uptake in the future and desired new aspects in the field of vaccine development will be highlighted. The goal of this update is to provide facts which may help to better implement the existing recommendations for influenza immunisation.

\section{Epidemiology and clinical characteristics}

Influenza viruses (orthomyxoviridae) can be divided into three major antigenic groups named types A, B and C. While natural infections with viral types $B$ and $C$ are restricted to human beings, type $\mathrm{A}$ infections also occur in birds, pigs and horses [39]. Type A viruses can be further subtyped according to genetic variability of their major virulence factors, i.e. haemagglutinin (HA) (designated $\mathrm{H} 1-\mathrm{H} 15)$ and neuraminidase (NA) (N1-N9) 


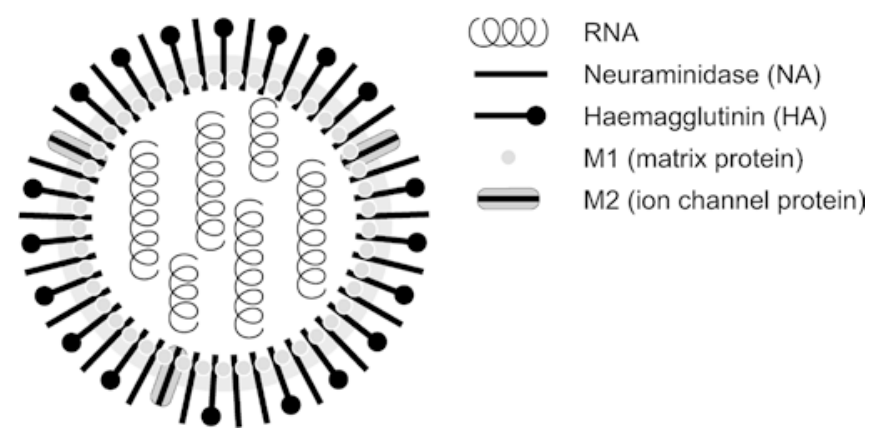

Fig. 1 Structure of influenza type A virus with HA and NA as major virulence factors
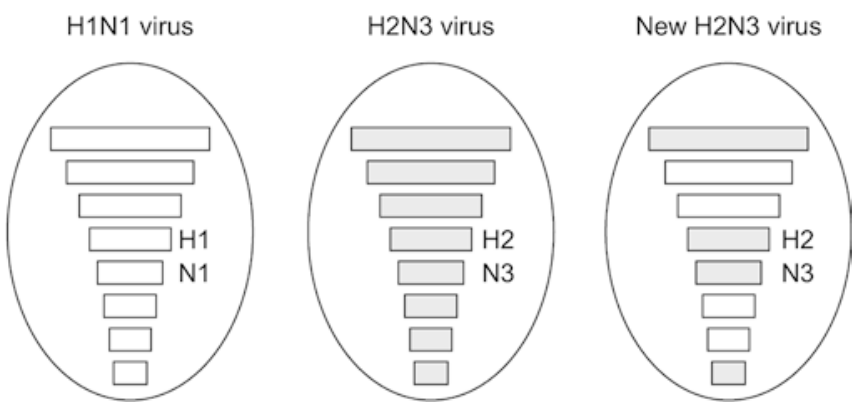

Fig. 2 Antigenic shift by re-assortment of different influenza A virus subtypes leading to a new subtype

antigens (Fig. 1) [39]. Point mutations (determined as antigenic drifts) which seem to occur by selective pressure, lead to antigenic changes within specific influenza virus $\mathrm{A}$ and $\mathrm{B}$ subtypes. They are responsible for yearly influenza epidemics, where type A and B viruses frequently co-circulate. In contrast, major antigenic changes can take place by means of re-assortment between gene segments when different human and animal influenza virus subtype A strains happen to infect a host at the same time (Fig. 2), as has been shown experimentally [56]. This results in an antigenic shift to a completely new subtype (e.g. from H1N1 to H2N3; Fig. 2) and can lead to pandemics, the last of which occurred in 1977 [25]. Since then, virus subtypes A/H1N1, A/H2N3 and B have been most prevalent. Influenza type $\mathrm{C}$ viruses occasionally cause local outbreaks. Their potential for epidemics is limited and they therefore play only a minor clinical role [14, 25, 39].

In the Northern hemisphere, outbreaks of influenza $\mathrm{A}$ and $\mathrm{B}$ occur during the cold season with peaks usually between December and February. Unfortunately, from an epidemiological and clinical point of view, other viruses such as respiratory syncytial virus (RSV) and parainfluenza virus also cause frequent respiratory infections in children with highest incidence rates during the winter season. Therefore, these infections frequently overlap with the influenza peak and this has complicated our appreciation of the true burden of influenza in the paediatric age group in the past. Fortunately, new diagnostic tools (such as PCR and rapid antigen tests applied to nasopharyngeal specimens) and increasing use of culture techniques have recently provided us with a much clearer picture of the aetiological role of influenza viruses in acute viral respiratory infections in children [42,57]. In a study from Finland, 683 children hospitalised in one university hospital were diagnosed with influenza between 1980 and 1999 [42]. Median age of patients with influenza A $(n=544)$ was 2.0 years compared to 4.2 years in those with influenza B $(n=139)$. Of these patients, $60 \%$ were boys and the vast majority $(75 \%)$ were previously healthy.

In a regional study from Northern Germany performed between 1996 and 2001, nasopharyngeal specimens of hospitalised children up to 16 years of age with respiratory infections were analysed by multiplex PCR [57]. Similar to the observations in Finland, children with influenza A $(n=122)$ had a median age of 2.1 years compared to 2.6 years in those with influenza B $(n=14)$. Incidences (per 100,000 children per year) were 53, 16 and 165 for influenza A, influenza B and RSV, respectively. The highest incidence rates for all three virus infections were recorded in children $0-5$ years of age (123, 30 and 453, respectively) with peaks in the 1st (RSV: 1563) and 2nd years of life (influenza A: 161). Although absolute numbers of cases were comparatively small, as in Finland, most of the children hospitalised for influenza were previously healthy $(79 \%)$. Nevertheless, relative risks for hospitalisation due to influenza A were significantly increased for children with asthma $(4.1 ; 95 \%$ CI: $1.7-10)$ and underlying cardiac conditions (9.8; 95\% CI: 4.3-23) [57].

The epidemiological role of influenza in children is multi-facetted. On the one hand, the high burden of disease leads to physician visits, interference with daily activities such as absence from school, and occasionally to serious complications. On the other hand, children have been found to be important multiplicators in the infectious circle of influenza. They are regularly affected early during the start of an epidemic and thus serve as the source of consequent infections in adult contacts $[13,50]$.

Classical influenza is a respiratory infection with sudden onset of fever, rhinitis, a non-productive, hacking cough, myalgia and malaise, which affects patients at all ages $[9,39]$. Interestingly, vomiting and diarrhoea can be the major symptoms in young infants, especially with influenza A virus infection [41,47]. Major complications of influenza, which frequently lead to hospitalization in previously healthy children, include febrile convulsions, bronchiolitis, pneumonia, croup and myositis [42,57]. In the Finnish study, otitis media (26\% and $19 \%$ of children with influenza A and B, respectively), febrile convulsions $(12 \%$ and $9 \%)$, pneumonia $(9 \%$ and $8 \%)$, and croup $(5 \%$ and $4 \%)$ were the most common complications [42]. Influenza virus infections have also been identified as a major cause of subsequent secondary bacterial infections [37,49]. Among these, acute otitis media, primarily due to Streptocococcus pneumoniae, plays a major role and best explains the significant use of 
antibiotics in children with influenza [2,42]. Moreover, pneumococcal pneumonia and invasive meningococcal infections have been closely associated with influenza virus infections $[30,38]$.

Children and adolescents with underlying chronic illnesses may suffer from significant deterioration when infected with influenza viruses. In children with intrinsic asthma, influenza virus infection can trigger exacerbations $[28,51,52]$. It has further been shown that influenza is responsible for about $13 \%$ of acute exacerbations of cystic fibrosis during the winter season [46]. Also, children with malignant diseases more frequently acquire influenza and their illnesses are more severe compared to healthy age-matched controls [27].

In conclusion, influenza virus type A and B infections are a frequent cause of hospitalisation in previously healthy children during the epidemic season. Children and adolescents with underlying illnesses are at even higher risk for hospitalisation due to influenza when compared to primary healthy controls. Based on epidemiological knowledge, preventive measurements in patients with underlying conditions are highly justified. Moreover, given the high burden of disease in primary healthy children, immunisation strategies beyond the current target groups at increased risk should be discussed.

\section{Prevention}

Immunisation

Today, several types of inactivated influenza vaccines are available: whole-cell, split, subunit and virosomal (Fig. 3). The selection of virus strains and the NA and $\mathrm{HA}$ antigens to be included in the vaccines is based on WHO recommendations. Laboratories throughout the world participate in the WHO global influenza surveillance system and constantly screen circulating influenza viruses for their antigenic constitution. Usually in February each year, the WHO recommends which three influenza strains should be used to formulate the vaccines for the coming season in the northern hemisphere. Reference strains will then be made available for vaccine manufacturers who have only a few months time for culture of the viruses, antigen purification, toxicity and immunogenicity analyses, mass production and finally distribution of the new vaccine.

The traditional whole virus vaccines demonstrated favourable immunogenicity results; however, due to their comparatively high reactogenicity, they are not licensed for use in children and have also been widely replaced in other age groups [60]. Compared to wholecell vaccines, "split" vaccines (consisting of pieces of virus as a consequence of disrupting the whole viruses with detergents) have a much better reactogenicity profile but also reduced immunogenicity, especially in young children [17]. Therefore, two doses of vaccine are recommended in children up to the age of 3 years (or

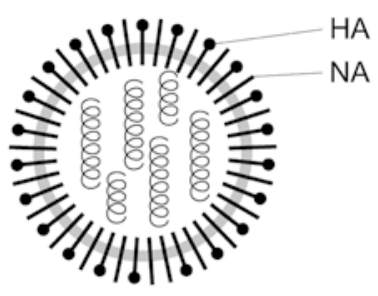

whole virus vaccine

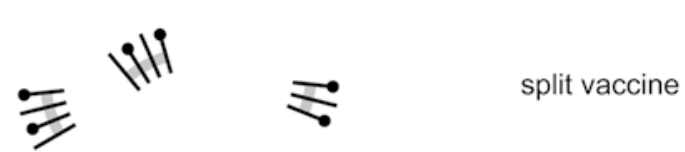

|||१९९ subunit vaccine

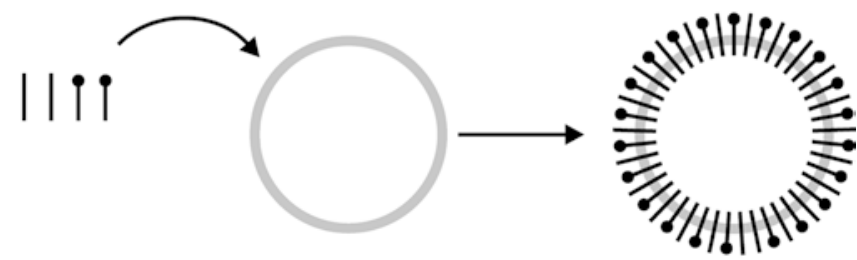

$\mathrm{NA}+\mathrm{HA}$ phospholipid structure virosome vaccine

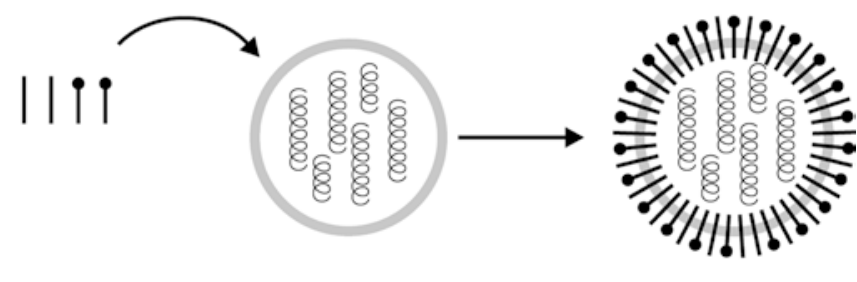

$\mathrm{NA}+\mathrm{HA} \quad$ donor strain $\quad$ CAIV-T

Fig. 3 Schematic composition of various influenza vaccines

older in some European countries and the United States) when immunised against influenza for the first time. "Subunit" vaccines have been further purified when compared to split vaccines and mainly contain HA and NA [29]. Safety and reactogenicity findings with subunit vaccines in children are favourable $[4,10]$. As with split vaccines, two doses within 4 to 8 weeks are required for young first time vaccinees. Split and subunit vaccines are widely available throughout Europe and many other countries worldwide.

A new class of influenza vaccine has recently been developed in Switzerland (Inflexal V). The vaccine consists of immunopotentiating reconstituted influenza virosomes, which are a combination of natural phospholipids, phospholipids deriving from influenza NA and HA and phophatidylcholine [15, 16,32]. The vaccine is trivalent containing three distinct virosome pools. Lot consistency and stability over prolonged periods of time (18 months at $5^{\circ} \mathrm{C}, 3$ months at $25^{\circ} \mathrm{C}$ and 28 days at $37^{\circ} \mathrm{C}$ ) have been demonstrated [32]. Clinical experience with the virosome influenza vaccine in children has 
recently been summarised [24]. Briefly, in four investigations a total of 127 children 1 to 12 years of age ( 80 healthy and 43 with cystic fibrosis) received virosome vaccine and tolerability $(n=127)$ and immunogenicity $(n=116)$ were evaluated. When compared to parallel groups receiving subunit vaccine, the immunogenicity requirements as defined by the EMEA, the European registration body, were fulfilled at similar rates; specific comparative antibody levels have not been published. Tolerability of virosome vaccine was similar to that of the subunit vaccine with $54 \%$ and $55 \%$ of vaccinees experiencing one or more local adverse events and $44 \%$ and $59 \%$ one or more systemic adverse event, respectively. Of note, favourable tolerability and no untoward immunological effects (CD4 + count and viral load) of the virosome vaccine have been obtained in a study of 23 HIV-positive children [64]. Inflexal $\mathrm{V}$ is currently licensed in 13 European countries: Austria, Denmark, Finland, Germany, Greece, Italy, the Netherlands, Portugal, Spain, Sweden, Switzerland (and Liechtenstein), and the United Kingdom (R. Hoos, Berna Biotech, Switzerland, personal communication).

Over the last two decades, a live-attenuated, re-assortant, cold-adapted trivalent influenza vaccine (CAIV$\mathrm{T})$ has been developed and studied intensively in all age groups including young children [14]. It contains structural genes from attenuated donor strains of types $\mathrm{A}$ and $\mathrm{B}$ and re-assorted $\mathrm{N}$ and $\mathrm{H}$ genes of currently circulating influenza viruses. Administration is by the intranasal route with a large-droplet spray $(0.25 \mathrm{ml}$ per nostril $)$, which makes this vaccine attractive especially, but not only, for children. The donor strains are also temperature sensitive ("cold adapted"): optimal viral replication occurs at $25^{\circ} \mathrm{C}$ (the approximate temperature in the nasal cavity) with virtually no replication at $37^{\circ} \mathrm{C}$ thus avoiding spread to the lower airways of the vaccinees. Immunological responses to this vaccine are pronounced and comprise not only stimulation of systemic but also of mucosal immunity. Therefore they resemble natural infection more than any other influenza vaccines [31]. Importantly, significant immune responses can be induced even in young infants if repetitive doses are used [7].

Efficacy of CAIV-T has been investigated in several studies in healthy children and adolescents (Table 1). In a pivotal study where 1602 children 15 to 71 months of age received one or two doses of CAIV-T 6 to 10 weeks apart, overall efficacy against culture-confirmed influenza virus infection was $93 \%$ (95\% CI 88-96) with rates of $89 \%$ and $94 \%$ for the one or two-dose schedules, respectively [3]. Specifically, after one and two doses, efficacy against influenza A/H3N2 was $87 \%(95 \% \mathrm{CI}$ : 47-97) and 96\% (90\%-99\%), respectively. Respective efficacy rates for influenza virus B were $91 \%(46 \%$ $99 \%$ ) and $91 \%(78 \%-96 \%)$. In the following year, efficacy against an antigenically drifted $\mathrm{H} 3 \mathrm{~N} 2$ virus type A was still $86 \%$. Results of this and other studies are summarised in Table 1. Studies in children and adolescents with underlying chronic illness (e.g. asthma) are currently being conducted. Of note, concerns have been expressed that repeated application of CAIV (which, like other influenza vaccines needs to be adapted each year) might lead to an impaired ability of replication due to induction of an immune response against core antigens of the master assortant [43]; However, clinical experience so far has not supported this theoretical concern [3, $12,18]$.

Tolerability of CAIV-T appears to be good. Mild upper respiratory tract symptoms such as rhinorrhoea and nasal congestion have been observed at rates of $10 \%$ above those after placebo on days 2 and 3 after nasal administration of the vaccine in children [2]. Short-lived and low-grade fever attributable to vaccine was observed only on day 2 of the first dose $(6.5 \%$ of vaccinees compared to $1.6 \%$ of placebo controls) as was decreased activity $(6.0 \%$ versus $2.1 \%)$. Sequential annual doses of CAIV-T in the same cohort were generally well tolerated with low rates of mild respiratory, gastrointestinal and systemic symptoms [45]. Data on tolerability of CAIV-T in children and adolescents with underlying illnesses are limited and mainly derive from studies in asthmatics or patients with cystic fibrosis [19, 33,54]. Further studies are currently being conducted in several European countries.

Shedding of CAIV-T has been found in nasopharyngeal secretions of vaccinees for up to 12 days [1]. This has raised concerns about the possibility of vaccine virus transmission to close contacts; however, evidence so far indicates that this does not appear to be a frequent event, if it occurs at all [61]. Further safety considerations with the use of CAIV-T are directed towards the possibility that "infection" with the vaccine virus might

Table 1 Efficacy Trials with CAIV-T in children and adolescents

\begin{tabular}{|c|c|c|c|c|}
\hline Reference & Study subjects $(n)$ & Age & Vaccine efficacy $(\%)$ & Comments \\
\hline [44] & $192 / 169$ & $3-18$ years & $\begin{array}{l}\text { A/H3N2; } 1 \text { dose: } 89 \% \\
\text { A/H1N } 1 ; 1 \text { dose: } 100 \%\end{array}$ & Bivalent vaccine without type B \\
\hline [3] & 1602 & 15-71 months & $\begin{array}{l}\text { A/H3N2; } 1 \text { dose: } 87 \% ; \mathrm{A} / \mathrm{H} 3 \mathrm{~N} 2 ; \\
2 \text { doses: } 96 \% ; \mathrm{B} ; 1 \text { dose: } 91 \% \text {; } \text {; } \\
2 \text { doses: } 91 \%\end{array}$ & $\begin{array}{l}\text { Pivotal efficacy trial which led to licensure } \\
\text { of the vaccine in the United States }\end{array}$ \\
\hline
\end{tabular}


pave the way for secondary bacterial infections, genetic and phenotypic alterations in the CAIV master virus, and re-assortment of CAIV with influenza wild type virus genes in co-infected vaccinees [43]. Experience so far lends no support for any of these concerns. However, careful post-licensure surveillance will definitely be warranted now that CAIV-T (Flumist) has been licensed for the first time in the United States for individuals 5 to 49 years of age for the influenza season 2003/2004.

Recently, a killed, nasally applied influenza vaccine had been developed using virosomal technology and Escherichia coli heat-labile toxin as a mucosal adjuvant and licensed in Switzerland [15]. Unfortunately, several cases of peripheral facial palsy were reported in vaccinees during the first season after licensure which lead to withdrawal of the vaccine from the market (press release by the Swiss Federal Health Office, September 14, 2001; http://www.bag.admin.ch/dienste/medien/2001/d/ 01091424.htm). An extensive safety study was performed the following year in several countries (unpublished) and resulted in termination of further production of this vaccine by the manufacturer (press release by Berna Biotech, June 6, 2002).

Although inactivated influenza whole-cell, split, subunit, and virosome influenza vaccines are produced by use of embryonated hen eggs, their application in children with allergy to ingested egg proteins (mainly yolk proteins, ovalbumin and ovomucoid) in general appears to be safe $[26,63]$. CAIV-T is produced using hen's egg allantoic fluid. Although this vaccine has not been studied thoroughly in children with allergy to egg proteins, no indication for hypersensitivity after nasal administration of the vaccine has been observed so far [43]. Nevertheless, the usual precautions for possible anaphylactic reactions should be applied as a routine measurement when immunising against influenza.

\section{Considerations on current influenza immunisation recommendations}

As can be seen in Table 2, there is currently significant heterogeneity with respect to which groups of children and adolescents are considered to be at increased risk from influenza in various European countries. This is surprising because the direct effects of influenza vaccination programmes based on a risk group strategy are obvious: morbidity and mortality can be reduced in those who have been immunised. However, since these individuals represent only a small minority of all children and adolescents, indirect effects in the sense of herd immunity cannot be achieved with such a strategy. Of note, several countries emphasise in their national guidelines that influenza immunisation should be considered for anyone with the desire to be protected from influenza, although these costs will not be covered by health insurance.

It has been shown that schools are the main source for spread of influenza virus at the beginning of each epidemic [13]. The Japanese experience, where influenza immunisation was mandatory for schoolchildren from 1977 to 1987, demonstrated that such an expanded immunisation programme not only prevents consequences of influenza (morbidity, absence from school) in the vaccinees themselves, but also indirectly protected other vulnerable segments of the population, i.e. the elderly and other high risk patients [50]. In my view, whether such a strategy, primarily aiming at providing herd immunity, would be acceptable for parents and physicians is doubtful for most European countries. At least as long as yearly injections would still be necessary, it is hard to believe that such a goal could be achieved, when it is difficult enough to convince parents of high risk patients about the benefits of influenza immunisation. Furthermore, any expanded influenza immunisation programme would need to be implemented on a voluntary basis because significant resistance against mandatory immunisations could be envisioned by parts of the populations of most if not all European countries today.

\section{Future challenges for influenza immunization}

Several difficult and not so difficult tasks related to influenza immunisation lie ahead of us.

First, and most importantly in my opinion, we need to better educate the current and the next generation of physicians about the threats of influenza and the most efficient way of prevention, i.e. immunisation. This educational process should start as early as in medical school and continue during postgraduate training in hospitals and continuously thereafter. In this respect, we recently performed a survey among physicians in our hospital where we assessed their attitudes towards immunisation against influenza [23]. Doubts about necessity $(56 \%)$ and efficacy $(32 \%)$ as well as concerns about side-effects $(24 \%)$ were the most frequently stated reasons by the approximately $50 \%$ of colleagues who had declined influenza immunisation. This example demonstrates existing barriers to influenza immunisation. Also, the public needs to be educated. Of importance is the fact that many people are unaware of the significant clinical difference between a common cold and "true" influenza. How can they appraise the value of the vaccine if they are not aware of the existence of complications of the disease?

Second, improvement of acceptance of influenza immunisation needs to be achieved in high risk patients. This goal can only be achieved in close link to the educational need stated above.

Third, better surveillance of morbidity and potential long-term sequelae in young infants is required in order to have a basis for the discussion of the issue of a general influenza immunisation strategy. At the same time, transmission patterns of influenza between children and adults should be studied.

Fourth, ways to avoid shortage of vaccine, which has been encountered recently, should be explored. 
Table 2 Recommendations for influenza immunisation in children and adolescents in selected European countries. Data are based on information obtained from the published immunisation recommendations of Austria (http://www.gesundheit.bmsg.gv.at), Germany (www.rki.de) and Switzerland and the following colleagues and members of the European Society for Paediatric Infectious Diseases (ESPID) as obtained in June 2003: H. Bogaerts (Belgium), V. Pellantova (Czech Republic), N. Valerius (Denmark), K. Zilmer (Estonia), H. Noyhnek (Finland), D. Gendrel and E. Grimprel (France), G. Syrogiannopoulos and M. Tsolia (Greece),
A. Haraldsson and S. Sigurdardottir (Iceland), S. Knowles (Ireland), S. Esposito and R.F. Schumacher (Italy), D. Gardovska (Latvia), V. Usonis (Lithuania), A. Berstad (Norway), A. Neto and L. Varandas (Portugal), N. Hawash and S. Yakushin (Russia), M. Cizman (Slovenia), F. Asensi (Spain), H. Heijbel (Sweden), R. de Groot and J. L. Kimpen (Netherlands), A. Finn and M. Sharland (UK). No information could be obtained for Bulgaria, Poland, Romania, Slovakia and the former Yugoslavia. "Yes": specifically indicated; "No": not indicated; "-": no official guideline exists

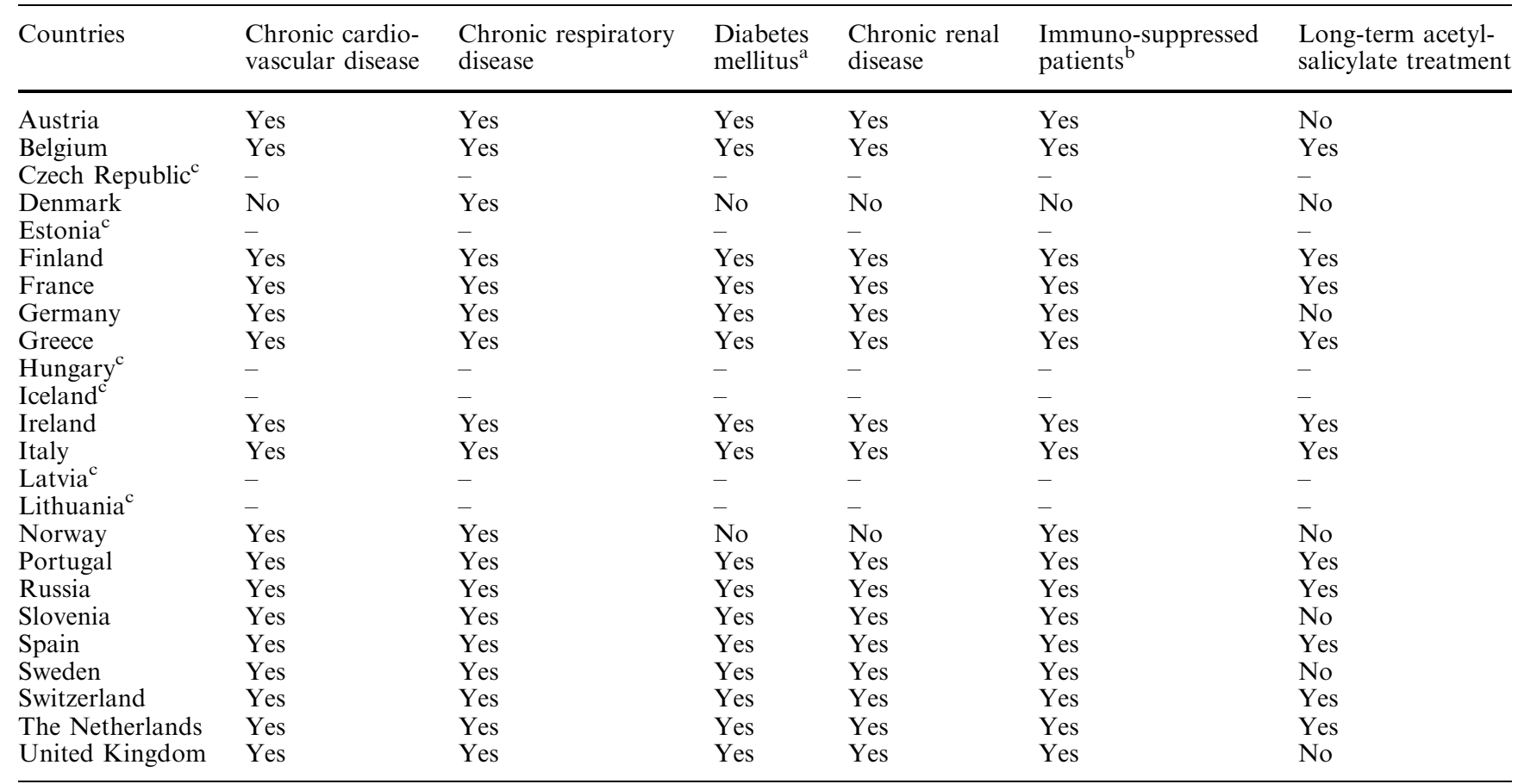

a'Explicitly mentioned or as part of "metabolic diseases" category; frequently limited to insulin-dependent diabetes mellitus

${ }^{\mathrm{b}}$ Explicitly including HIV-positive patients in some but not all countries; frequently including patients with haemoglobinopathies leading to functional asplenia

Currently, each dose of vaccine requires one hen's egg. Research should focus on the development of cell culture techniques for influenza vaccine strain production to overcome the current production limitations in terms of vaccine quantities.

Fifth, immunising pregnant women against influenza should be evaluated more intensively. This could not only decrease the risk for complications attributable to influenza in pregnant women themselves but would also potentially protect their infants via transplacental transfer of specific IgG antibodies for the first few months of their lives - a period where hospitalisation rates for influenza are highest [35].

Finally, the ultimate goal would be the development of a vaccine with efficacy beyond the next influenza season. Naturally, this would require detection of protective antigens which are not subject to regular mutations (drifts) and thus do not allow the virus to evade from the host's specific immune responses. Progress in genome sequencing has opened the door to this promising area of research.
${ }^{\mathrm{c} D e s p i t e}$ lack of official guidelines, influenza immunisations are performed on individual physicians' decisions and/or are supported by national paediatric societies in these countries

\section{Chemoprophylaxis}

In the recent past, the introduction of a new class of antiviral drugs, the NIs, has supplemented our armament to fight influenza [8]. In contrast to Amantadin, which blocks the ion channels (M2 protein) of influenza virus type A (Fig. 1) and has been only available for treatment of children with influenza type A but not type B infections for many years, NI are efficacious against types A and B. They can be used for treatment and prophylaxis [48]. They function by interfering selectively with the NA of influenza virus types A and B and thus inhibit the release of new viruses from infected cells (Fig. 4). Use of NIs may induce resistance in some influenza virus strains. However, these resistant strains have been shown to be defective in virulence and less contagious when compared to susceptible strains [20].

So far, two NIs have been licensed in Europe and other parts of the world. Zanamivir (Relenza) is insufficiently resorbed when given orally and therefore must be administered as a powder by inhalation. 
Fig. 4 Infection of respiratory cells by influenza virus, replication and release of new virus particles. Interference with release of viruses by NIs

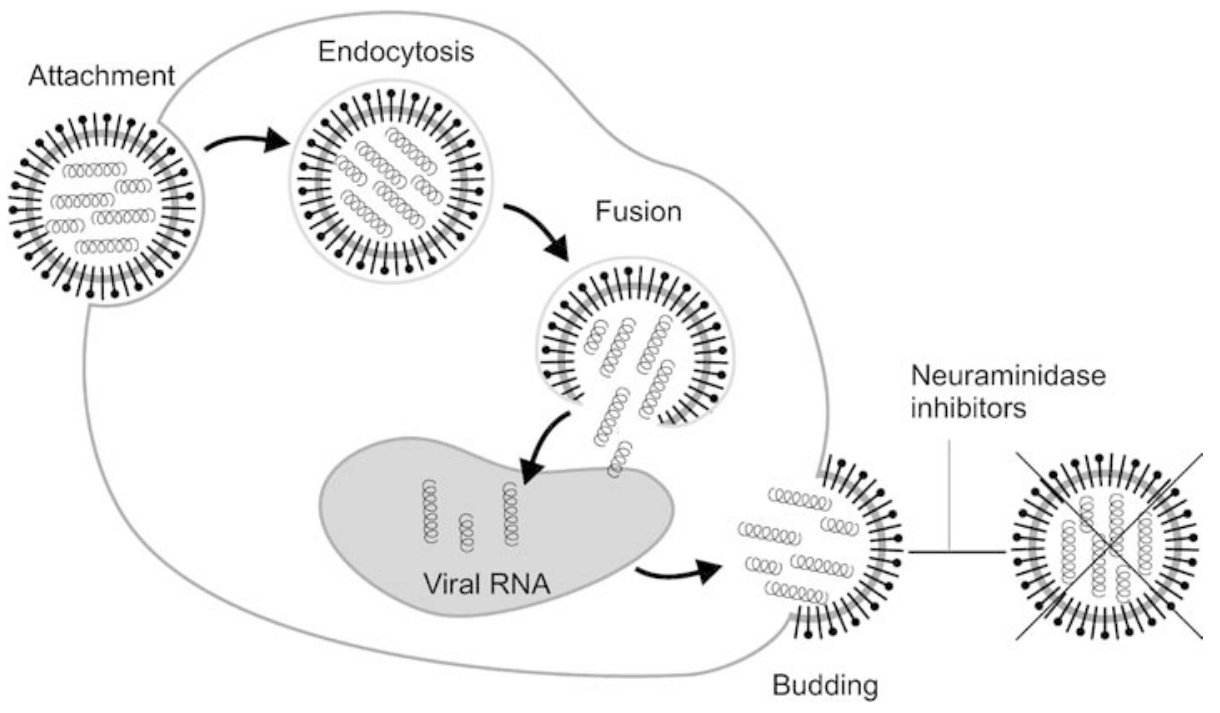

Doses of $5 \mathrm{mg}$ are administered with a special device called a "diskhaler". In most countries, Zanamivir has only been licensed for treatment of influenza in individuals 12 years of age and older but not for prophylaxis, although its preventive efficacy has been shown in studies in adults and children older than 5 years [22,34]. In Switzerland, Zanamivir has been licensed for treatment (5 years of age onwards) and prophylaxis (12 years of age onwards) of influenza for up to 1 month duration.

In contrast to Zanamivir, Oseltamivir (Tamiflu) has an excellent bioavailability after oral administration as a capsule or liquid suspension. The dosage is weight dependent and ranges from 30 to $75 \mathrm{mg}$. Based on available efficacy data, Oseltamivir has been licensed for treatment ( 1 year of age and older) and prophylaxis (from 13 years of age onwards) of influenza infections. For prevention of influenza, Oseltamivir can be given once daily during the influenza season and its tolerability has been shown for up to 6 consecutive weeks of administration. For post-exposure prophylaxis, once daily use for 7 days is recommended. However, for prevention of influenza in children and adolescents, the experience with this drug is limited. In one study, Oseltamivir demonstrated an efficacy of $82 \%$ when given once daily for 10 days as post-exposure prophylaxis to children who had been exposed to a sibling with influenza [21]. Probably because of the relatively high costs, clinical use of NIs is currently limited to a few countries, mainly Japan, the United States and some in Europe [53].

To my knowledge, the only guidelines in Europe for the use of NIs in high risk paediatric patients (i.e. those for whom influenza immunisation is recommended) have been developed by the Committee for Infectious Diseases and Vaccines of the German Academy for Paediatrics [11]. They are similar to those formulated by the United States Advisory Committee on Immunization Practices [6]. According to the German Committee, the use of Oseltamivir should be considered for prevention during epidemics of influenza under the following circumstances (note: age dependent licensure restrictions, which may differ from country to country, should be observed):

1. If influenza vaccine has not been administered due to a contraindication (use for up to 6 weeks)

2. If no influenza vaccine is available for prophylaxis (use for up to 6 weeks)

3. If influenza vaccine has only been administered after the onset of the epidemic and the period until full vaccine protection can be assumed is to be covered (use for 10 to 14 days)

4. If influenza vaccine has not been administered and exposure to a household member with suspected or proven influenza has occurred (use for 7 days)

5. If the influenza epidemic is caused by a mutated virus different from the current vaccine strains, irrespective of the patient's vaccination status (use for up to 6 weeks)

\section{Conclusions}

Influenza is known to be a frequent cause of respiratory tract infections in the paediatric age group. Further efforts are still needed to increase the knowledge among physicians and the public about the significant burden of disease in children and adolescents caused by influenza viruses. Moreover, increased educational activities about the benefits of prevention of influenza are necessary to better implement existing immunisation recommendations which would improve the health of our patients. These activities should be augmented by innovative research aiming at more efficient manufacturing technologies and new vaccines with prolonged efficacy. Finally, antiviral drugs like NIs can reasonably complement but not substitute our current influenza immunisation strategies. 


\section{References}

1. Belshe RB, Van Voris LP (1984) Cold-recombinant influenza $\mathrm{A} /$ California/10/78 (H1N1) virus vaccine (CR-37) in seronegative children: infectivity and efficacy against investigational challenge. J Infect Dis 149: 735-740

2. Belshe RB, Mendelman PM, Treanor J, King J, Gruber WC, Piedra P, Bernstein DI, Hayden FG, Kotloff K, Zangwill K, Iacuzio D, Wolff M (1998) The efficacy of live attenuated, coldadapted, trivalent, intranasal influenza virus vaccine in children. N Engl J Med 338: 1405-1412

3. Belshe RB, Gruber WC, Mendelman PM, Cho I, Reisinger K, Block SL, Wittes J, Iacuzio D, Piedra P, Treanor J, King J, Kotloff K, Bernstein DI, Hayden FG, Zangwill K, Yan L, Wolff M (2000) Efficacy of vaccination with live attenuated, cold-adapted, trivalent, intranasal influenza virus vaccine against a variant (A/Sydney) not contained in the vaccine. J Pediatr 136: 168-175

4. Bernstein DI, Cherry JD (1983) Clinical reactions and antibody responses to influenza vaccines. A comparison of split or subunit vaccines in children and young adults. Am J Dis Child 137: 622-626

5. Bridges CB, Fukuda K, Uyeki TM, Cox NJ, Singleton JA (2002) Prevention and control of influenza. Recommendations of the Advisory Committee on Immunization Practices (ACIP). MMWR Recomm Rep 51(RR-3): 1-31

6. Centers for Disease Control and Prevention (2003) Prevention and control of influenza. Recommendations of the Advisory Committee on Immunization Practices (ACIP). MMWR Recomm Rep 52 (RR-8): 1-36

7. Clements ML, Makhene MK, Karron RA, Murphy BR, Steinhoff MC, Subbarao K, Wilson MH, Wright PF (1996) Effective immunization with live attenuated influenza A virus can be achieved in early infancy. J Infect Dis 173: 44-51

8. Cooper NJ, Sutton AJ, Abrams KR, Wailoo A, Turner D, Nicholson KG (2003) Effectiveness of neuraminidase inhibitors in treatment and prevention of influenza A and B: systematic review and meta-analyses of randomised controlled trials. BMJ 326: 1235

9. Couch RB (2000) Prevention and treatment of influenza. N Engl J Med 343: 1778-1787

10. Daubeney P, Taylor CJ, McGaw J, Brown EM, Ghosal S, Keeton BR, Palache B, Kerstens R (1997) Immunogenicity and tolerability of a trivalent influenza subunit vaccine (Influvac) in high-risk children aged 6 months to 4 years. Br J Clin Pract 51: $87-90$

11. Deutsche Akademie für Kinderheilkunde und Jugendmedizin, Kommission für Infektionskrankheiten und Impfungen (Bartmann P, Heininger U, Huppertz HI, Kinet M, Korenke GC, Schick KH) (2003) Stellungnahme zur Verwendung von Neuraminidasehemmern zur Therapie bzw. Prophylaxe der Influenza bei Kindern und Jugendlichen. Monatsschr Kinderheilkd 151: 110-112

12. Edwards KM, Dupont WD, Westrich MK, Plummer WD Jr, Palmer PS, Wright PF (1994) A randomized controlled trial of cold-adapted and inactivated vaccines for the prevention of influenza A disease. J Infect Dis 169: 68-76

13. Glezen WP (1996) Emerging infections: pandemic influenza. Epidemiol Rev 18: 64-76

14. Glezen WP (2002) Influenza vaccination for healthy children. Curr Opin Infect Dis 15: 283-287

15. Glück R (2001) Pre-clinical and clinical investigation of the safety of a novel adjuvant for intranasal immunization. Vaccine 20[Suppl 1]: S42-S44

16. Glück R, Mischler R, Durrer P, Furer E, Lang AB, Herzog C, Cryz SJ Jr (2000) Safety and immunogenicity of intranasally administered inactivated trivalent virosome-formulated influenza vaccine containing Escherichia coli heat-labile toxin as a mucosal adjuvant. J Infect Dis 181: 1129-1132
17. Groothuis JR, Levin MJ, Rabalais GP, Meiklejohn G, Lauer BA (1991) Immunization of high-risk infants younger than 18 months of age with split-product influenza vaccine. Pediatrics 87: $823-828$

18. Gruber WC, Campbell PW, Thompson JM, Reed GW, Roberts B, Wright PF (1994) Comparison of live attenuated and inactivated influenza vaccines in cystic fibrosis patients and their families: results of a 3-year study. J Infect Dis 169: 241247

19. Gruber WC, Belshe RB, King JC, Treanor JJ, Piedra PA, Wright PF, Reed GW, Anderson E, Newman F (1996) Evaluation of live attenuated influenza vaccines in children 6-18 months of age: safety, immunogenicity, and efficacy. J Infect Dis 173: 1313-1319

20. Gubareva LV, Kaiser L, Hayden FG (2000) Influenza virus neuraminidase inhibitors. Lancet 355: 827-835

21. Hayden F, Belshe R, Villanueva C, Lanno R, Small I, Hughes C, Dutkowski R (2002). Oral oseltamivir prevents the spread of influenza between children and households (abstract). 42nd ICAAC,September 2002, San Diego, V-244

22. Hayden FG, Gubareva LV, Monto AS, Klein TC, Elliot MJ, Hammond JM, Sharp SJ, Ossi MJ (2000) Inhaled zanamivir for the prevention of influenza in families. Zanamivir Family Study Group. N Engl J Med 343: 1282-1289

23. Heininger U, Bachler M, Schaad UB (2003) Attitudes of pediatricians regarding influenza self-immunization: a survey in a Swiss university children's hospital. Pediatr Infect Dis J 22: 391-394

24. Herzog C, Metcalfe IC, Schaad UB (2002) Virosome influenza vaccine in children. Vaccine 20[Suppl 5]: B24-B28

25. Hilleman MR (2002) Realities and enigmas of human viral influenza: pathogenesis, epidemiology and control. Vaccine 20: 3068-3087

26. James JM, Zeiger RS, Lester MR, Fasano MB, Gern JE, Mansfield LE, Schwartz HJ, Sampson HA, Windom HH, Machtinger SB, Lensing S (1998) Safe administration of influenza vaccine to patients with egg allergy. J Pediatr 133: 624-628

27. Kempe A, Hall CB, MacDonald NE, Foye HR, Woodin KA, Cohen HJ, Lewis ED, Gullace M, Gala CL, Dulberg CS et al (1989) Influenza in children with cancer. J Pediatr 115: 33-39

28. Kondo S, Abe K (1991) The effects of influenza virus infection on FEV1 in asthmatic children. The time-course study. Chest 100: $1235-1238$

29. Laver WG, Webster RG (1976) Preparation and immunogenicity of an influenza virus hemagglutinin and neuraminidase subunit vaccine. Virology 69: 511-522

30. Makras P, Alexiou-Daniel S, Antoniadis A, Hatzigeorgiou D (2001) Outbreak of meningococcal disease after an influenza B epidemic at a Hellenic Air Force recruit training center. Clin Infect Dis 33: e48-e50

31. Mendelman PM, Cordova J, Cho I (2001) Safety, efficacy and effectiveness of the influenza virus vaccine, trivalent, types A and $\mathrm{B}$, live, cold-adapted (CAIV-T) in healthy children and healthy adults. Vaccine 19: 2221-2226

32. Mischler R, Metcalfe IC (2002) Inflexal V a trivalent virosome subunit influenza vaccine: production. Vaccine 20[Suppl 5]: B17-B23

33. Miyazaki C, Nakayama M, Tanaka Y, Kusuhara K, Okada K, Tokugawa K, Ueda K, Shibata R, Nishima S, Yamane N, Maassab HF (1993) Immunization of institutionalized asthmatic children and patients with psychomotor retardation using live attenuated cold-adapted re-assortment influenza $\mathrm{A}$ $\mathrm{H} 1 \mathrm{~N} 1, \mathrm{H} 3 \mathrm{~N} 2$ and $\mathrm{B}$ vaccines. Vaccine 11: 853-858

34. Monto AS, Robinson DP, Herlocher ML, Hinson JM Jr, Elliott MJ, Crisp A (1999) Zanamivir in the prevention of influenza among healthy adults: a randomized controlled trial. JAMA 282: 31-35

35. Munoz FM, Englund JA (2001) Vaccines in pregnancy. Infect Dis Clin North Am 15: 253-271 
36. Neuzil KM, Dupont WD, Wright PF, Edwards KM (2001) Efficacy of inactivated and cold-adapted vaccines against influenza A infection, 1985 to 1990: the pediatric experience. Pediatr Infect Dis J 20: 733-740

37. Neuzil KM, Zhu Y, Griffin MR, Edwards KM, Thompson JM, Tollefson SJ, Wright PF (2002) Burden of interpandemic influenza in children younger than 5 years: a 25 -year prospective study. J Infect Dis 15 185: 147-152

38. O'Brien KL, Walters MI, Sellman J, Quinlisk P, Regnery H, Schwartz B, Dowell SF (2000) Severe pneumococcal pneumonia in previously healthy children: the role of preceding influenza infection. Clin Infect Dis 30: 784-789

39. Olshaker JS (2003) Influenza. Emerg Med Clin North Am 21: 353-361

40. Pachucki CT, Lentino JR, Jackson GG (1985) Attitudes and behavior of health care personnel regarding the use and efficacy of influenza vaccine. J Infect Dis 151: 1170-1171

41. Paisley JW, Bruhn FW, Lauer BA, McIntosh K (1978) Type A2 influenza viral infections in children. Am J Dis Child 132: 34-36

42. Peltola V, Ziegler T, Ruuskanen O (2003) Influenza A and B virus infections in children. Clin Infect Dis 36: 299-305

43. Pfleiderer M, Lower J, Kurth R (2001) Cold-attenuated live influenza vaccines, a risk-benefit assessment. Vaccine 20: 886894

44. Piedra PA (1991) Influenza vaccines. Semin Pediatr Infect Dis 2: $140-146$

45. Piedra PA, Yan L, Kotloff K, Zangwill K, Bernstein DI, King J, Treanor J, Munoz F, Wolff M, Cho I, Mendelman PM, Cordova J, Belshe RB (2002) Safety of the trivalent, coldadapted influenza vaccine in preschool-aged children. Pediatrics 110: 662-672

46. Pribble CG, Black PG, Bosso JA, Turner RB (1990) Clinical manifestations of exacerbations of cystic fibrosis associated with nonbacterial infections. J Pediatr 117: 200-204

47. Price DA, Postlethwaite RJ, Longson M (1976) Influenza virus A2 infections presenting with febril convulsions and gastrointestinal symptoms in young children. Clin Pediatr (Phila) 15: 361-367

48. Prober CG (2002) Antiviral therapy for influenza virus infections. Semin Pediatr Infect Dis 13: 31-39

49. Rafei K (2002) Influenza virus vaccines in children and their impact on the incidence of otitis media. Semin Pediatr Infect Dis 13: 129-133

50. Reichert TA, Sugaya N, Fedson DS, Glezen WP, Simonsen L, Tashiro M (2001) The Japanese experience with vaccinating schoolchildren against influenza. N Engl J Med 344: 889-896
51. Roldaan AC, Masural N (1982) Viral respiratory infections in asthmatic children staying in a mountain resort. Eur J Respir Dis 63: $140-150$

52. Smits AJ, Hak E, Stalman WA, van Essen GA, Hoes AW, Verheij TJ (2002) Clinical effectiveness of conventional influenza vaccination in asthmatic children. Epidemiol Infect 128: 205-211

53. Stöhr K (2003) Preventing and treating influenza. BMJ 326 : $1223-1224$

54. Tanaka Y, Ueda K, Miyazaki C, Nakayama M, Kusuhara K, Okada K, Tokugawa K, Shibata R, Nishima S, Yamane N, Massab HF (1993) Trivalent cold recombinant influenza live vaccine in institutionalized children with bronchial asthma and patients with psychomotor retardation. Pediatr Infect Dis J 12: 600-605

55. Watanakunakorn C, Ellis G, Gemmel D (1993) Attitude of healthcare personnel regarding influenza immunization. Infect Control Hosp Epidemiol 14: 17-20

56. Webster RG, Campbell CH, Granoff A (1973) The "in vivo" production of "new" influenza viruses. 3. Isolation of recombinant influenza viruses under simulated conditions of natural transmission. Virology 51: 149-162

57. Weigl JA, Puppe W, Schmitt HJ (2002) The incidence of influenza-associated hospitalizations in children in Germany. Epidemiol Infect 129: 525-533

58. Weingarten S, Riedinger M, Bolton LB, Miles P, Ault M (1989) Barriers to influenza vaccine acceptance. A survey of physicians and nurses. Am J Infect Control 17: 202-207

59. Woods M, Robke JT (2003) Removing hospitalization as a barrier to immunization. Clin Infect Dis 36: 673-674

60. Wright PF, Thompson J, Vaughn WK, Folland DS, Sell SH, Karzon DT (1977) Trials of influenza A/New Jersey/76 virus vaccine in normal children: an overview of age-related antigenicity and reactogenicity. J Infect Dis 136[Suppl]: S731S741

61. Wright PF, Okabe N, McKee KT Jr, Maassab HF, Karzon DT (1982) Cold-adapted recombinant influenza A virus vaccines in seronegative young children. J Infect Dis 146: 71-79

62. Yassi A, Murdzak C, Cheang M, Tran N, Aoki FY (1994) Influenza immunization: knowledge, attitude and behaviour of health care workers. Can J Infect Control 9: 103-108

63. Zeiger RS (2002) Current issues with influenza vaccination in egg allergy. J Allergy Clin Immunol 110: 834-840

64. Zuccotti GV, Cucchi C, Sala D, Giovannini M (2002) Immunogenicity and safety of a virosomal influenza vaccine in HIV-infected children. Acta Paediatr 91: 486 